\title{
Proceedings of the GRAPPA 2016 Retreat
}

\author{
Deepak R. Jadon, Dafna D. Gladman, Philip J. Mease, Oliver FitzGerald, Vinod Chandran, \\ Niti Goel, Cheryl F. Rosen, Walter P. Maksymowych, Christopher T. Ritchlin, Alexis Ogdie, \\ Laura C. Coates, Alberto Cauli, Enrique R. Soriano, M. Elaine Husni, Willemina Campbell, \\ Valderilio F. Azevedo, Kristina Callis Duffin, April W. Armstrong, Alice B. Gottlieb, \\ Arthur Kavanaugh, Amit Garg, and Philip S. Helliwell
}

\begin{abstract}
In advance of its 2016 annual meeting, members of the steering committee of the Group for Research and Assessment of Psoriasis and Psoriatic Arthritis (GRAPPA) convened for a strategic planning meeting. The purpose of this advance meeting was to review the work of GRAPPA since its inception in 2003, ascertain and review the current priorities of the group, and devise a strategy for proceeding. The key accomplishments of GRAPPA to date, priorities and objectives for the next 5 years, and goals and opportunities for the GRAPPA committees were discussed. GRAPPA has a responsibility and commitment to patients, its members, and partners to innovate, inspire, and improve knowledge and the ability to care for people with psoriasis and psoriatic arthritis. (J Rheumatol 2017;44:668-73; doi:10.3899/jrheum.170141)
\end{abstract}

Key Indexing Terms:

PSORIATIC ARTHRITIS PSORIASIS EDUCATION RESEARCH GRAPPA

The Group for Research and Assessment of Psoriasis and Psoriatic Arthritis (GRAPPA) steering committee convened immediately before the GRAPPA Annual Meeting 2016 in Miami, Florida, USA, for an inaugural strategic planning meeting. A brief history of GRAPPA and its key initiatives has recently been published ${ }^{1}$, and it informed discussions at this meeting.

\section{Key Accomplishments of GRAPPA}

Each steering committee member proposed what they
From the Rheumatology Research Unit, Cambridge University Hospitals UK National Health Service (NHS) Foundation Trust, Cambridge; UK National Institute for Health Research Clinical Lecturer, Leeds Institute of Rheumatic and Musculoskeletal Medicine, University of Leeds, Leeds; Bradford Hospitals NHS Foundation Trust, Bradford, UK; Department of Medicine, Division of Rheumatology, University of Toronto, Toronto Western Hospital, Krembil Research Institute; Division of Dermatology, Toronto Western Hospital, University of Toronto, Ontario; University of Alberta, Edmonton, Alberta, Canada; Rheumatology Research, Swedish Medical Center and University of Washington School of Medicine, Seattle, Washington; Advisory Services, QuintilesIMS, Denver, Colorado; Duke University School of Medicine, Durham, North Carolina; Division of Allergy, Immunology, and Rheumatology, University of Rochester Medical Center, Rochester; New York Medical College, Valhalla; Hofstra Northwell School of Medicine, New Hyde Park, New York, New York; Medicine and Epidemiology, Perelman School of Medicine, University of Pennsylvania, Philadelphia, Pennsylvania; Cleveland Clinic, Cleveland, Ohio; University of Utah, Salt Lake City, Utah; University of Southern California, Los Angeles; University of California at San Diego, San Diego, California, USA; Newman Clinical Research Professor, Department of Rheumatology, St. Vincent's University Hospital; Conway Institute for Biomolecular Research, University College Dublin, Dublin, Ireland; Division of Rheumatology, University of Cagliari, Cagliari, Italy, Sección Reumatología, Servicio de Clínica Médica, Hospital Italiano de Buenos Aires, Buenos Aires, Argentina; Federal University of Paraná, Curitiba, Brazil.

As part of the supplement series GRAPPA 2016, this report was reviewed internally and approved by the Guest Editors for integrity, accuracy, and consistency with scientific and ethical standards.

D.R. Jadon, MBBCh, MRCP, PhD, Director, Rheumatology Research Unit, Cambridge University Hospitals NHS Foundation Trust; D.D. Gladman, $M D, F R C P C$, Professor of Medicine, University of Toronto, and Senior Scientist, Krembil Research Institute, Toronto Western Hospital; P.J. Mease, MD, Rheumatology Research, Swedish Medical Center and
University of Washington School of Medicine; O. FitzGerald, MD, FRCPI, FRCP $(U K)$, Newman Clinical Research Professor, Department of Rheumatology, St. Vincent's University Hospital, and Conway Institute for Biomolecular Research, University College Dublin; V. Chandran, MBBS, MD, DM, PhD, Department of Medicine, Division of Rheumatology, University of Toronto, Toronto Western Hospital, Krembil Research Institute; N. Goel, MD, Patient Research Partner, Vice President, Advisory Services, QuintilesIMS, and Adjunct Assistant Professor, Duke University School of Medicine; C.F. Rosen, MD, FRCPC, Division of Dermatology, Toronto Western Hospital, University of Toronto; W.P. Maksymowych, $M D, F R C P(C)$, Professor of Medicine, University of Alberta; C.T. Ritchlin, MD, MPH, Professor of Medicine, Division of Allergy, Immunology, and Rheumatology, University of Rochester Medical Center; A. Ogdie, MD, Assistant Professor of Medicine and Epidemiology, Perelman School of Medicine, University of Pennsylvania; L.C. Coates, $M B C h B, P h D, U K$ National Institute for Health Research Clinical Lecturer, Leeds Institute of Rheumatic and Musculoskeletal Medicine, University of Leeds; A. Cauli, MD, PhD, Division of Rheumatology, University of Cagliari; E.R. Soriano, MD, MSC, Sección Reumatología, Servicio de Clínica Médica, Hospital Italiano de Buenos Aires; M.E. Husni, MD, MPH, Cleveland Clinic; W. Campbell, BEd LLB, Patient Research Partner, Toronto Western Hospital; V.F. Azevedo, MD, PhD, Adjunct Professor of Rheumatology, Federal University of Paraná; $K$. Callis Duffin, MD, University of Utah; A.W. Armstrong, $M D, M P H$, University of Southern California; A.B. Gottlieb, MD, PhD, Professor of Dermatology, New York Medical College; A. Kavanaugh, MD, Professor of Medicine, University of California at San Diego; A. Garg, MD, Hofstra Northwell School of Medicine; P.S. Helliwell, DM, PhD, FRCP, University of Leeds, and Bradford Hospitals NHS Foundation Trust.

Address correspondence to Dr. P.S. Helliwell, Leeds Institute of Rheumatic and Musculoskeletal Medicine, University of Leeds, 2nd Floor, Chapel Allerton Hospital, Harehills Lane, Leeds LS7 4SA, UK. E-mail:p.helliwell@leeds.ac.uk 
considered to have been GRAPPA's top 3 accomplishments since inception. In unranked order, 8 themes emerged:

1. International collaborations that led to the Classification Criteria for Psoriatic Arthritis (CASPAR) study group for psoriatic arthritis (PsA) ${ }^{2}$, more collaborative work between rheumatologists and dermatologists, and the fostering of an inclusive culture within GRAPPA;

2. Education, including the global symposia organized by GRAPPA, in particular in collaboration with other organizations, e.g., the Spondyloarthritis Research and Treatment Network (SPARTAN); trainee symposia and presentations at GRAPPA annual meetings; the educational content of the GRAPPA Website, particularly the training videos; and the GRAPPA textbook of psoriatic disease;

3. Treatment recommendations, especially the advent of treat-to-target for PsA and psoriasis, and how GRAPPA treatment recommendations influence commissioners/funders of clinical services;

4. Research projects, including the development of the CASPAR criteria, and the PsA Biomarkers for Joint Damage (BioDAM) project;

5. Patient research partners (PRP) bringing patient groups and clinicians together through a culture of open communication and exchange of ideas from members, including PRP participation at GRAPPA meetings, and co-publication of papers with patients as lead authors;

6. Outcome measures, including the International Dermatology Outcome Measures (IDEOM) mentorship initiative, the development and validation of outcome measures through collaboration with the Outcome Measures in Rheumatology (OMERACT) and IDEOM groups, and the GRAPPA Composite Exercise (GRACE) project to develop new composite measures;

7. Professional development, e.g., opportunities for trainees' involvement and professional growth through GRAPPA;

8. Reputation, e.g., the respect of GRAPPA by clinicians, patients, regulatory authorities, and industry, including awareness and knowledge of psoriatic disease research through annual publication of the proceedings of GRAPPA meetings in The Journal of Rheumatology.

\section{Priorities for the Next 5 Years}

Three key objectives were identified as priorities for GRAPPA over the next 5 years.

1. What do we want members, partners, and others to be noticing about GRAPPA?

GRAPPA should cultivate attributes for which it is already being positively noticed, e.g., publications, group membership, and its reputation as a functional interdisciplinary international organization. Recognition can be built through:

- Patient service organizations and individual patients, including PRP.
- $\quad$ Regulatory organizations, e.g., the US Food and Drug Administration, Rockville, Maryland (FDA), and the European Medicines Agency (EMA).

- Healthcare service providers and payors, e.g., insurance companies and large healthcare institutions.

- International nongovernment organizations such as the World Health Organization (WHO) that collaborate with organizations such as OMERACT and the International Federation of Psoriasis Associations, leading to the establishment of World Psoriasis Day.

- $\quad$ The pharmaceutical industry.

- International clinical groups such as the American College of Rheumatology (ACR), Canadian Dermatology Association, European League Against Rheumatism (EULAR), American Academy of Dermatology (AAD), European Academy of Dermatology and Venereology (EADV), Pan American League of Associations for Rheumatology (PANLAR), Asia Pacific League of Associations for Rheumatology, International Psoriasis Council (IPC), Assessment of Spondyloarthritis international Society (ASAS), and SPARTAN.

GRAPPA should build on its presence at international dermatology and rheumatology conferences, e.g., the EADV, $\mathrm{AAD}, \mathrm{ACR}$, and EULAR annual meetings, and target the clinical needs of community-based rheumatologists and dermatologists who provide clinical care and are less involved in research.

2. What do we want members, partners, and others to be saying about GRAPPA?

For its members, GRAPPA should develop its role as a leading place for professional and career development through online educational material, leaflets for patients, and regional symposia. The GRAPPA Website should be enhanced to offer better resources, e.g., links to treatment recommendations and patient resources. GRAPPA should build on its growing reputation for efficiently driving projects forward while minimizing unnecessary bureaucracy. GRAPPA can address the unmet need for greater collaboration in basic science research, using well-characterized patient cohorts and biomaterial. Given that GRAPPA has worldwide membership, more active involvement should be encouraged from geographic regions where English is not the primary language. Continuing to host helpful, enjoyable meetings was considered a priority, with a broader number of venues for GRAPPA symposia to engage members wishing to be more involved and encourage recruitment of new members.

GRAPPA should aspire to be the leading global clinical and research group for psoriasis and PsA. By complementing rather than competing with similar organizations, GRAPPA should leverage its unique strengths to add value in areas of unmet need, e.g., clinical recommendations related to diagnosis and treatment, patient outcomes, and actionable biomarkers and new treatment targets.

Personal non-commercial use only. The Journal of Rheumatology Copyright @ $\odot 2017$. All rights reserved 
For patients, GRAPPA should be seen as an organization that bridges the gap between dermatologists and rheumatologists, thereby allowing more comprehensive integrated care. GRAPPA should facilitate the involvement of PRP in local research grant applications, partly to improve the success of grant applications, but more importantly to ensure research is addressing areas considered a patient priority. Improving the functionality of the GRAPPA Website and developing it as a portal to collate patient resources, such as those provided by the Psoriasis and Psoriatic Arthritis Alliance, ACR, DermNet.nz, and RheumInfo.com, might be useful for patients.

\section{What do we want members, partners, and others to value about GRAPPA?}

GRAPPA's goal should be to lead in defining research directions in psoriatic disease, the ethics and conduct of clinical studies and trials, expertise in regulatory issues and outcome measures, biomedical and translational research, refining and defining treatment targets in psoriatic disease, developing educational resources, and being the go-to organization for commissioners/funders. GRAPPA should also take a leading role in fostering the development of the next generation of investigators globally through the funding of research grants and establishment of formal mentoring programs. GRAPPA will need to further cultivate its collaborative spirit to achieve these goals.

\section{Six Key Objectives Going Forward}

Six key objectives for GRAPPA are identified (Table 1). GRAPPA has a responsibility to inspire and motivate the interest of the clinical and research community in PsA and psoriasis. Attaining high organizational strength and communication will require robust governance, succession planning, improved communication, administrative thoroughness, financial planning, branding through name recognition and marketing, and a membership strategy that balances financial reality with growth, regionalization, and engagement of members. Achievement of these objectives would identify GRAPPA as an exemplar for other similarly sized organizations.

The current GRAPPA membership is estimated to be about 650 . Attendance at the annual meeting has grown each year, with 80 delegates in 2008, 350 delegates in 2014 when the meeting was combined with the annual SPARTAN

Table 1. Six key objectives for the Group for Research and Assessment of Psoriasis and Psoriatic Arthritis over the next 5 years.

\begin{tabular}{ll} 
1. & Professional development and networking \\
2. & Education \\
3. & Assessment and management \\
4. & Collaboration \\
5. & Organizational strength and communication \\
6. & Research \\
\hline
\end{tabular}

meeting, and 200 delegates in 2016 . While a small yearly membership fee would raise some capital and might encourage greater member participation, this may be at the expense of losing valuable membership. The success of CASPAR (30 centers contributing) and GRAPPA treatment recommendations (40 centers contributing) owes much to GRAPPA membership being free and inclusive. The efforts of members who tirelessly promote GRAPPA and psoriatic disease research regionally without recognition are very much appreciated by GRAPPA and should not be overlooked.

Regionalization may be helpful for both membership and education. Representation at the GRAPPA annual meeting from regional groups may help disseminate GRAPPA's efforts and initiatives to unengaged audiences. GRAPPA symposia in South America, Asia, Africa, Europe, and Australasia may further facilitate member participation and education. Engagement of GRAPPA with regional psoriatic groups, such as the British Psoriatic Arthritis Consortium (Brit-PACT), could be a vehicle to aid regionalization and maintain a unified approach to the management and research of psoriasis and PsA.

Agreement on equitable membership fees across the diverse economies from which GRAPPA members currently contribute will be a challenge. Stratifying membership rates for non-trainees, trainees, industry partners, and patients poses a further hurdle because administrating such a complex system may not result in a net surplus of membership fees. An alternative system proposed and discussed was to have a fellow member status for those who regularly contribute to GRAPPA initiatives and events, and an associate member status for members who use resources, but do not directly contribute.

\section{Unmet Needs in the Care of Patients with Psoriatic Disease}

Advancing the care of individuals with psoriasis and PsA requires GRAPPA to focus on 4 key unmet needs. First, to increase early diagnosis of psoriasis and PsA through the development of early referral strategies and the promotion of patient registries. Second, better tools for assessing patients must be developed and existing tools more thoroughly validated. Third, GRAPPA needs to spearhead the evaluation of treatment modalities available for psoriasis and PsA, and finally, develop internationally recognized treatment recommendations. Although impressive gains in treatment response have taken place over the last decade in psoriatic disease, disease remission particularly in PsA is not common, emphasizing the need for more effective therapies at an affordable price. To tackle these unmet needs, GRAPPA must support clinical research, mediate collaborative research efforts, and identify priority areas for research that will maximize the fruits of these endeavors to areas most important to patients. A cornerstone of the strategy will be greater PRP involvement by engaging with patient advocacy organizations, networking

Personal non-commercial use only. The Journal of Rheumatology Copyright @ 2017 . All rights reserved. 
forums, and other professional organizations to identify priority areas for research and disseminate the completed works so that they are translated to the bedside. Collaboration with regulatory agencies, payors, and the biopharmaceutical industry will need to be woven into this strategy.

\section{Cross Cutting: Goals and Opportunities for the GRAPPA Committees}

For each of the 7 GRAPPA committees, the steering committee identified at least 1 major goal for the next 5 years, key activities to undertake or continue over the coming year to achieve this goal, and potential cross-cutting themes or engagement opportunities.

\section{Professional development and networking}

The establishment of a trainee-mentor network would allow trainees to identify mentors with skills and experience that might aid their own professional development in a symbiotic way. A further, perhaps overlooked need, is mentorship for young GRAPPA members who have completed their formal rheumatology or dermatology training. A clinical and academic career requires lifelong learning, and such individuals might also benefit from the expertise of a mentor. Such a network might be strengthened through the provision of sessions on how to be a mentor teach-the-teachers and train-the-trainers programs being good examples of this.

For a number of years, the GRAPPA annual meeting has embedded trainee sessions in its core program. Trainee feedback has consistently praised these sessions because they provide a platform for trainees to disseminate their data, refine their presentation skills, and receive internal peer review by the GRAPPA delegates. The inclusion of such trainee sessions of PsA research in larger international annual meetings, e.g., ACR, AAD, or PANLAR, might further these rewards.

Regional GRAPPA meetings should encourage the involvement of local clinicians to promote professional development such as presentation skills and critiquing scientific literature. Gaining Continuous Medical Education (CME) or Continuous Professional Development (CPD) accreditation for GRAPPA meetings would increase incentives to attend.

\section{Education}

GRAPPA is a natural leader to provide educational resources for PsA and psoriasis. In a technically innovative way, some existing projects require refinement, and a number of new initiatives need be started. GRAPPA should aim to improve skills, not just knowledge.

GRAPPA's online resources currently include e-learning modules, a slide deck, and videos. The video project would be enhanced by adding vendors, skin phenotypes, certificates of module completion, and diversification of the languages in which the videos are broadcast. The provision of non-English content might in turn increase membership from the non-English-speaking parts of the world, which are currently underrepresented in GRAPPA. The opportunity for retrospectively broadcasting keynote speeches or sessions from GRAPPA annual and regional meetings in the form of Webinars would provide greater access to GRAPPA members unable to travel to these meetings. Another opportunity might be to disseminate knowledge and generate interest in psoriatic disease through a regular image of the week, question of the week, or case of the week, perhaps through weekly e-mails, Tweets, or downloadable apps. Additional benefits may be CME points and attracting traffic to the hosting GRAPPA Website.

The GRAPPA textbook of psoriatic disease is an example of cross-specialty international collaboration. GRAPPA might consider producing or endorsing existing patient leaflets on psoriatic disease.

A strategy is needed to stimulate interest in psoriatic disease. This might be achieved through better partnership with the large international conferences, e.g., EADV, AAD, ACR, and EULAR. GRAPPA symposia within the AAD, rather than the current adjacent sessions, might attract more attention. Technology Entertainment Design (TED)-style talks, increasingly popular in other industries, could inspire and motivate innovation for psoriatic disease, on current treatments, future treatments, and controversies.

Finally, a multidisciplinary team educating each other could improve the care of patients with psoriatic disease. Participants may benefit from a rheumatologist teaching dermatology trainees how to examine for arthritis, and a dermatologist teaching rheumatology trainees about the different phenotypes of psoriasis.

\section{Assessment and management}

GRAPPA can be a leader in assessment and management by engaging with other organizations. Treat-to-target (T2T) remains a key objective, and requires analysis of data from clinical trials and observational studies, and surveying of existing members' opinions through a Delphi process. The ultimate goal would be to achieve consensus within the membership, and the publication of T2T recommendations.

GRAPPA can also lead in establishing core outcome measures in psoriatic disease as part of the OMERACT initiative. This will require a systematic literature review, survey of GRAPPA members, and attainment of a consensus.

An unmet need, on which much work has already been done but no consensus reached, is the ideal strategy for screening for PsA in dermatology clinics and the community. Collaboration with the National Psoriasis Foundation might help drive this forward.

Finally, the GRAPPA treatment recommendations for PsA published in 2016 will require updating for the projected 2020 target year.

Personal non-commercial use only. The Journal of Rheumatology Copyright $\subset$ 2017. All rights reserved. 


\section{Collaboration}

GRAPPA can be strengthened only by collaboration with international organizations such as the PANLAR, International League of Associations for Rheumatology, EULAR, ACR, OMERACT, IDEOM, AAD, and EADV; disease-specific organizations such as the ASAS, SPARTAN, and IPC; joining forces with previously nonpartnered groups such as the WHO and BRIT-PACT; and regulatory groups such as the EMA and FDA. Such relationships could be formalized through contracts containing shared or agreed research agendas, rather than simply a memorandum of understanding. Doing so might help maintain ties despite changes in leadership over time, formalizing agreements such as complimentary registration, facilitating collaborative grant applications, and organizing educational symposia.

\section{Organizational strength and communication (people)}

For GRAPPA to be a successful organization, it is important to engage and communicate with members, other interested parties, and healthcare providers - hospital-based physicians, community-based physicians, specialist/research nurses, and physicians' assistants. Branding can be improved by increased visibility (e.g., GRAPPA Website improvements), encouraging inclusion of the GRAPPA slide deck with associated GRAPPA logo in presentations by members, and a higher profile for the GRAPPA brand in publications that GRAPPA has supported. Greater allocation of resources and funds will be required to improve Website functionality, including the search function of slide decks, collating images from members, disseminating a case of the week; publishing textbooks and in journals; hiring staff to create and disseminate regular newsletters; and to use the full potential of social media, e.g., Twitter, Facebook, and LinkedIn.

\section{Organizational strength and communication (infra- structure)}

Organizational strength consists of 3 key facets: governance, finance, and administration. It is quite notable that GRAPPA's governance rules have not changed in the last 10 years, reflecting their relevance and acceptance in the organization. However, complacency should be avoided, and GRAPPA steering committee member governance priorities should remain at the forefront for the coming years, particularly the need to clarify the membership structure and achieve growth and financial stability. Future membership of the steering committee may mandate attendance at a certain number of meetings and proven activity in a subcommittee(s). The appointment of a new bookkeeper in recent months has helped GRAPPA's administrative capacity. However, there is a degree of uncertainty in GRAPPA finances, with peaks and troughs based on events, invoicing, and payments. An endowment fund may be considered, but would first require designating money each year to build the fund. Other possibilities are a charitable donation system on the GRAPPA Website, or fundraising events such as walks, half-marathons, or gala dinners. The introduction of membership fees has both advantages and disadvantages. Stable finances and active membership clarification must be balanced against the cost of administering membership and potentially losing charity status. It will be a challenge to determine an equitable membership fee across countries with different economic positions, and across different grades of member seniority. A principle of GRAPPA is to be inclusive and international, and a membership fee may therefore be counterproductive.

\section{Research}

GRAPPA has funded several projects, resulting in a significant number of published studies; this work should continue. One of the major goals for GRAPPA over the next 5 years is to establish a GRAPPA Research Collaboration Network, which would agree on common longitudinal projects, collecting data from inception and retrospective cohorts, and including patient-reported outcome measures (PROM), clinical examination indices, and biomaterial. Establishing standard operating procedures (SOP) for biomaterial collection would be an important task for the network.

Providing research support for trainees and young investigators remains a central mission of GRAPPA. GRAPPA should provide funds for pilot grants that will be awarded annually on a competitive basis. A review committee consisting of dermatologists, rheumatologists, methodologists, and PRP should be identified and convene yearly to review the proposals.

Underpinning this effort would be the establishment of a GRAPPA BioResource, a research network for sharing biomaterials, clinical data, and imaging through a virtual repository. GRAPPA would not store the biomaterials or secure grants for specific research studies. Instead, individual network members would devise and fund their own research studies, but apply to the BioResource for clinical data, imaging data, and/or biomaterial that may complement their own resources as part of analytical studies or replication studies. Members would also notify the network about which clinical, imaging, and biomaterials they would be willing to contribute to the BioResource. The biomaterials would be kept by the individual network member, who could share with another network member once an application to the network steering committee for a research study has been approved. The existing GRAPPA-BioDAM would be incorporated into the BioResource.

The GRAPPA network steering committee would review requests for materials as part of a scientific advisory board to help direct precious biomaterial and data to priority research areas within psoriatic disease. Co-authorship on publications by contributing centers would also need to be incorporated into the strategy. The first step will be to

Personal non-commercial use only. The Journal of Rheumatology Copyright @ 2017 . All rights reserved. 
establish interest and expertise within the GRAPPA membership for such a network and BioResource, after which a steering committee can be established to coordinate the logistics, define the research agenda, and attract funds to support the network and BioResource. Harmonization of data collection and SOP for imaging and biomaterial collection will be crucial, and consideration must be given for setting up the network to clinical trial standards. The network could provide seed money for well-defined exploratory studies using the collected data, and pump-priming for larger externally funded projects. Such a network would further GRAPPA's engagement with academic, patient, and industry partners.

In time, through the platform such a network would provide, trainees and other members could participate in exchange programs to learn new skills, e.g., performing ultrasound-guided synovial biopsy or skin biopsies; studies of genomics, proteomics, metabolomics, etc.; bioinformatics; and using clinical examination indices in the research setting. There may be scope for GRAPPA to fund these fellowships through research bursaries.

\section{DISCUSSION}

GRAPPA has flourished as an organization dedicated to the research and management of psoriasis and PsA, and has a responsibility and commitment to individuals with psoriatic disease, its members, and partners to continue this effort. GRAPPA aspires to innovate and inspire others to collaborate so that our understanding and ability to care for people with psoriatic disease continue to improve. The GRAPPA steering committee has set out priorities in a strategic plan that will help the organization grow over the next 5 to 10 years.

\section{ACKNOWLEDGMENT}

GRAPPA acknowledges the contribution to this work of Pamela Love and her team, and Ruth Nicholson.

\section{REFERENCES}

1. Gladman DD, Mease PJ, Boehncke WH, Helliwell PS, Callis Duffin K. Presidential round table: a report from the GRAPPA Annual Meeting. J Rheumatol 2016;43:986-9.

2. Taylor W, Gladman D, Helliwell P, Marchesoni A, Mease P, Mielants H; CASPAR Study Group. Classification criteria for psoriatic arthritis: development of new criteria from a large international study. Arthritis Rheum 2006;54:2665-73. 